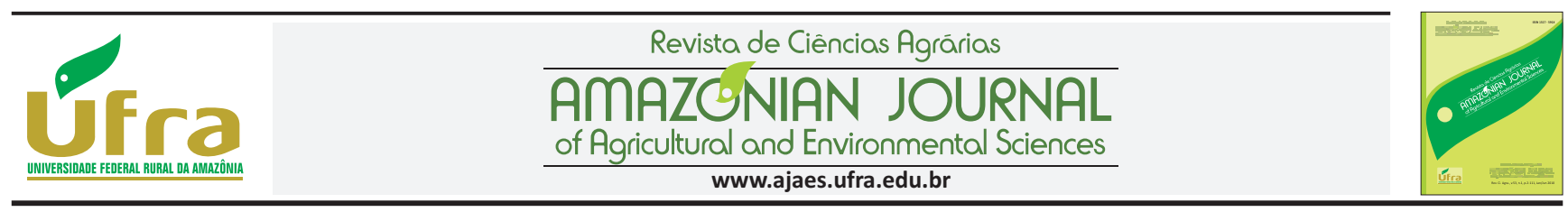

AUTORES:

Johnatt Allan Rocha de Oliveira ${ }^{1}$

Ana Vânia Carvalho

Débora Kono Taketa Moreira $^{3}$

Luiza Helena da Silva Martins $^{4}$

${ }^{1}$ UEPA, Trav. Eneas Pinheiro, 2626, 66095-100, Belém, Pará, Brasil.

${ }^{2}$ Embrapa Amazônia Oriental, Av Dr. Enéas Pinheiro, s/n, Marco, 66095-100, Belém, Pará, Brasil.

${ }^{3}$ Instituto Federal de Educação, Ciência e Tecnologia do Sul de Minas Gerais. Praça Tiradentes, 416, 37576-000, Inconfidentes, MG, Brasil.

${ }^{4}$ UNICAMP. Rua Roxo Moreira, 13083-000, Campinas, SP, Brasil.

Recebido: 11/02/2010 Aprovado: 17/11/2010

AUTOR CORRESPONDENTE:

Johnatt Allan Rocha de Oliveira E-mail: johnattrocha@yahoo.com.br

PALAVRAS-CHAVE:

Theobroma grandiflorum,

Hidrocolóides

Fruta estruturada.

KEY WORDS:

Theobroma grandiflorum, Hydrocolloids,

Restructured fruits.

\section{Elaboração e caracterização de estruturado obtido de polpa concentrada de cupuaçu}

\section{The development and evaluation of a restructured from of concentrated cupuassu pulp}

Resumo: O objetivo deste trabalho foi elaborar um estruturado a partir de polpa concentrada de cupuaçu, avaliando-se o efeito da combinação de gelatina e pectina de baixa metoxilação nas características do gel da fruta. Realizou-se, na polpa de cupuaçu e nos estruturados obtidos, análises de composição centesimal, atividade de água, pH, acidez titulável, sólidos solúveis $\left(\mathrm{Brix}^{\circ}\right)$, açúcares totais e redutores e teor de vitamina $\mathrm{C}$, além da análise sensorial dos produtos finais. Os resultados mostram que é possível estabelecer um procedimento tecnológico para a produção de fruta estruturada com alto teor de polpa, variando entre 78,24 a 81,43\%. Optou-se pela formulação 3, como aquela de possível aplicação em escala industrial, pois foi a que apresentou melhor aceitação sensorial. De maneira geral, todas as formulações apresentaram-se como um produto final de textura firme, sem pegajosidade e saudável, tendo em vista seus teores de vitamina C (240,9 a $\left.247,3 \mathrm{mg} \mathrm{kg}^{-1}\right)$ e fibras $(3,23$ a 3,90\%), considerados significativos do ponto de vista nutricional.

Abstract: The aim was to develop a structured form of concentrated pulp cupuassu, evaluating the effect of the combination of gelatin and low methoxy pectin on the characteristics of the fruit gel. Analysis of chemical composition, water activity, $\mathrm{pH}$, acidity, soluble solids $\left(\mathrm{Brix}^{\circ}\right)$, total and reducing sugars and vitamin $C$ was carried out on the pulp and structured cupuassu obtained, in addition to sensory analysis of the end products. The results show that it is possible to establish a technical procedure for production of structured fruit with high pulp cupuassu ranging from 78.24 to $81.43 \%$. We opted for the third formulation for possible application on an industrial scale, because it was more acceptable in sensory terms. Generally, all the formulations provided a final product of firm texture without stickiness, and beneficial for health, in view its vitamin C (240,9 to $247,3 \mathrm{mg} \mathrm{kg}^{-1}$ ) and fiber content (3, 23 to $3.90 \%$ ). 


\section{Introdução}

O cupuaçu é uma das mais importantes frutas tipicamente amazônicas, e o Estado do Pará é o principal produtor (LOPES, 2000; BUENO et al., 2002; FREIRE et al., 2009). O fruto vem conquistando o mercado consumidor de outras regióes que não a sua de origem, com os mais diversos produtos que podem ser obtidos a partir de sua polpa (CALZAVARA et al., 1987; VILLACHICA, 1996; LOPES, 2000).

O cupuaçu, assim como outras frutas desenvolvidas em regiões tropicais, apresenta problemas especiais na manipulação pós-colheita. A umidade e as temperaturas elevadas, comumente encontradas nessas regiões, agravam a deterioração da fruta por bolores (VILLACHICA, 1996; ALZAMORA et al, 1992; SANTOS, 2003). Uma alternativa, na tentativa de reduzir as causas de deterioração pós-colheita, seria o controle da atividade de água do tecido da fruta, obtido por processamentos específicos (FREITAS, 1999; COSTA et al., 2003) que além de aumentar o tempo de permanência da fruta com qualidade, estimulariam a elaboração de novos produtos (SANTOS, 2003). Dentre as técnicas de processamento, a estruturação de polpa de frutas representa uma inovação na área de alimentos, com resultados bastante promissores (BELLARD, 1995).

O conceito de alimento estruturado ou "designed food" ou "engineered food" refere-se a alimentos que são delineados de acordo com um planejamento, geralmente empregando-se matérias-primas de baixo custo, oriundas de frutas que se encontram fora de classificação para comercialização in natura ou excedentes de produção durante o período de safra, ou mesmo subprodutos de outras indústrias como, por exemplo, recortes de carnes, frango ou pescado, proteínas animais ou vegetais, em muitos casos, utilizando-se hidrocoloides (FIZMAN, 1992; GRIZOTTO et al., 2006).

Estruturados de frutas são produtos obtidos por desidratação do purê devidamente formulado para obtenção de um produto nutritivo, com boa textura, sabor e cor (GRIZOTTO et al., 2004). São produzidos a partir de géis de pectato ou alginato com sais de cálcio ou alumínio, açúcares, corantes, aromatizantes (naturais ou artificiais) e pequenas porcentagens de polpa de fruta, variando de 0 a $41 \%$ do peso do produto (GRIZOTTO et al., 2005). Os hidrocolóides neste caso irão atuar como agentes de união, facilitando o corte e retendo umidade, fatores estes que contribuirão para a melhoria da textura (FIZMAN, 1992; GRIZOTTO et al., 2005).
O purê é normalmente espalhado em bandejas a uma espessura de cerca de $5 \mathrm{~mm}$ e seco até que uma consistência mastigável e agradável se desenvolva (BELLARD, 1995; SANTOS, 2003). Esses produtos podem ser utilizados na formulação de produtos de confeitaria ou alimentos congelados, ou mesmo consumidos na forma em que se apresentam, como um confeito, similarmente às barras de frutas (fruit bar), também conhecidas como "couro" de frutas (leather fruit) (VIJAYANAND et al., 2000).

O objetivo deste trabalho foi elaborar três estruturados de fruta com diferentes concentrações de gelatina, a partir de polpa concentrada de cupuaçu e caracterizar os produtos finais quanto aos seus atributos físico-químicos e sensoriais.

\section{Material e Métodos}

Foi utilizada polpa congelada de cupuaçu, adquirida no mercado local da cidade de Belém-PA. A polpa foi concentrada em rota-evaporador à temperatura de $50{ }^{\circ} \mathrm{C}$ até atingir $15^{\circ} \mathrm{Brix}$. Os hidrocoloides utilizados na preparação dos estruturados foram gelatina 180 Bloom (Rebière Gelatinas Ltda, Brasil) e pectina de baixa metoxilação (CPKelco, Brasil). Foram utilizados como coadjuvantes tecnológicos no processo de estruturação, fosfato de cálcio bibásico anidro (Synth, Brasil), glicerol (Vetec, Brasil) e sacarose refinada comercial (União, Brasil).

Para todas as formulações foram utilizados $100 \mathrm{~g}$ de polpa concentrada, a qual foi adicionada de um supressor de atividade de água: glicerol $(10 \% \mathrm{em}$ relação à polpa) e, em função do teor de sólidos solúveis determinado, calculou-se a quantidade de sacarose comercial suficiente para elevar o teor de sólidos para $65^{\circ}$ Brix ( $5 \mathrm{~g}$ de sacarose). A estruturação foi conduzida na polpa previamente aquecida a $60{ }^{\circ} \mathrm{C}$ em banho-maria, durante $15 \mathrm{~min}$. À polpa aquecida e sob agitação em misturador da marca Yamato Labo - Stirrer, modelo L_-35, com intensidade de agitação correspondente à posição cinco do reostato do aparelho, foram adicionados os ingredientes referentes às três formulações estudadas: Formulação $1-5 \%$ de gelatina, $2 \%$ de pectina de baixa metoxilação e $0,8 \%$ de fosfato de cálcio bibásico; Formulação 2-8\% de gelatina, 2\% de pectina de baixa metoxilação, 0,8\% de fosfato de cálcio bibásico; Formulação $3-10 \%$ de gelatina, $2 \%$ de pectina de baixa metoxilação, $0,8 \%$ de fosfato de cálcio bibásico. Para todas as formulações foi necessária a prévia dissolução do fosfato de cálcio bibásico em $2 \mathrm{~mL}$ de 
água destilada antes de sua adição.

Para a moldagem dos estruturados foram utilizadas placas de petri de $8 \mathrm{~mm}$ de altura e $50 \mathrm{~mm}$ de diâmetro, com capacidade para $20 \mathrm{~g}$ da amostra, aproximadamente. As frutas estruturadas foram mantidas sob refrigeração a $10^{\circ} \mathrm{C}$, durante $24 \mathrm{~h}$, para completarem a geleificação. Após isso, os estruturados foram cortados, com auxílio de um cortador de aço inoxidável em formato de corações, de aproximadamente $3,5 \mathrm{~cm}$ de diâmetro, e, em seguida, submetidos à secagem em estufa com circulação de ar a $50{ }^{\circ} \mathrm{C}$, por um período de 6 horas. O efeito da secagem sobre os estruturados foi avaliado visualmente, observandose principalmente a pegajosidade ao toque e a resistência ao corte.

O fluxograma para produção de fruta estruturada é apresentado na Figura 1.

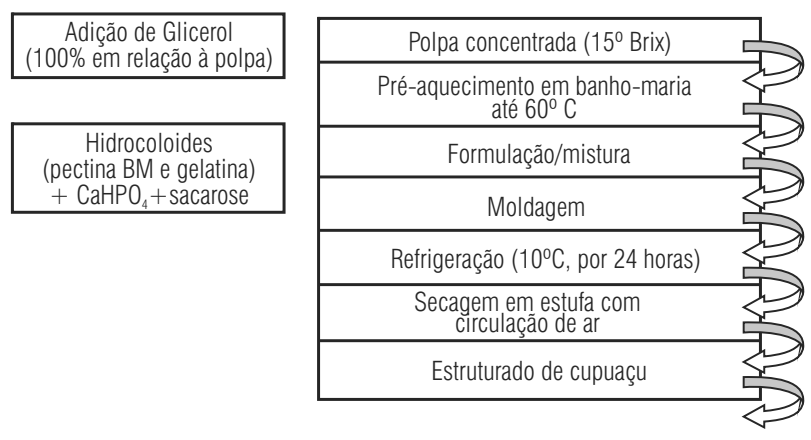

Figura 1. Fluxograma para o processamento do estruturado obtido de polpa concentrada de cupuaçu.

As análises realizadas para a caracterização físico-química da polpa de cupuaçu "in natura" e das três formulações de estruturado de cupuaçu foram as seguintes: atividade de água (obtida por medida direta em analisador de atividade de água Decagon, modelo Pawkit, Pullman, EUA), sólidos solúveis totais, determinados com refratômetro óptico Atago, modelo N1- 0-32 ํix (AOAC, 1997), acidez titulável (AOAC, 1997), pH (AOAC, 1997), umidade por estufa a $105^{\circ} \mathrm{C}(\mathrm{AOAC}, 1997)$, cinzas, por calcinação a $550^{\circ} \mathrm{C}(\mathrm{AOAC}, 1997)$, proteínas (AOAC, 1997), lipídios (BLIGH; DYER, 1959), fibras totais, pelo método de detergência (GOERING; VAN SOEST,1970), açúcares totais e redutores, por titulação de oxirredução (LANE; EYNON, 1984) e vitamina C por titulação, segundo o método no 43.065 da AOAC (1984), modificado por Benassi (1990).

A avaliação sensorial dos estruturados de cupuaçu foi realizada por 30 provadores não treinados, funcionários, estagiários e visitantes da Embrapa
Amazônia Oriental. Empregou-se o teste de aceitação com escala hedônica estruturada de 9 pontos (9=gostei muitíssimo; 1 =desgostei muitíssimo) (STONE; SIDEL, 1985). As amostras foram apresentadas aos potenciais consumidores à temperatura ambiente, em pratos plásticos descartáveis codificados com números aleatórios de 3 dígitos e avaliadas quanto à aparência, aroma, textura, sabor e impressão global. Na mesma ficha o provador ainda opinava quanto à sua intenção de compra em relação a cada uma das amostras.

O experimento foi conduzido em delineamento inteiramente casualizado, com cada provador recebendo uma única vez cada amostra. A nota recebida para cada amostra foi medida, utilizando-se régua milimetrada, realizou-se a soma das notas e posterior retirada das médias. Os resultados das características físico-químicas foram submetidos à análise de variância e as médias, quando significativas, comparadas pelo teste de Tukey a 5\% de probabilidade, com auxílio do programa SAS 8.0 (Statistical Analysis System).

\section{Resultados e Discussão}

Os resultados obtidos para caracterização físico-química da polpa e dos estruturados de cupuaçu encontram-se com valores aceitáveis, com destaque para o estruturado 3 (Tabela 1). Tais resultados estão de acordo com os relatados por Calzavara (1987), cujos valores foram: teores de $81,3 \%$ de umidade, $1,7 \%$ de proteínas, $1,6 \%$ de lipídios, $0,5 \%$ de fibras e 0,7\% de cinzas. Em estudo realizado por Freire et al. (2009), os autores caracterizaram polpas congeladas de cupuaçu e encontraram valores que variaram de 0,74 a 0,76\% de proteína, 0,54 a 1,33\% de lipídios e 0,82 a 4,84 $\mathrm{mg} \mathrm{kg}^{-1}$ de vitamina C. Já Bueno et al. (2002) encontraram para a polpa de cupuaçu teor de $258 \mathrm{mg} \mathrm{kg}^{-1}$ de vitamina $\mathrm{C}$, próximo ao encontrado neste trabalho, que foi de $192,3 \mathrm{mg} \mathrm{kg}^{-1}$.

Para as análises de $\mathrm{pH}$, sólidos solúveis, acidez titulável e atividade de água da polpa de cupuaçu observou-se valores de 3,62, 12,33 ${ }^{\circ} \mathrm{Brix}, 2,29 \%$ e 0,98 , respectivamente. Estes valores foram semelhantes aos observados por Costa et al. (2003), que encontraram valores para o pH de 3,34, sólidos solúveis de $12,5^{\circ} \mathrm{Brix}$, acidez titulável de 2,27\% e atividade de água de 0,98. Estes resultados ainda confirmam que a polpa de cupuaçu estudada estava dentro dos padrões de identidade e qualidade (PIQs) estabelecidos para a polpa de cupuaçu, segundo o Ministério da Agricultura e do Abastecimento (2000). 
Tabela 1 Caracterização físico-química da polpa e dos estruturados de cupuaçu, em base úmida.

\begin{tabular}{|c|c|c|c|c|}
\hline \multirow{2}{*}{ Determinações } & \multicolumn{4}{|c|}{ Amostras } \\
\hline & Polpa & Formulação1 & Formulação 2 & Formulação 3 \\
\hline Atividade de água & $0,980,001$ & $0,600,001 a$ & $0,570,001 b$ & $0,550,002 c$ \\
\hline $\mathrm{pH}$ & $3,620,02$ & $3,950,03 b$ & $3,950,02 b$ & $3,990,02 a$ \\
\hline Sólidos solúveis $\left(B r i x^{\circ}\right)$ & $12,330,05$ & $58,670,06 b$ & $60,000,04 a b$ & $61,330,05 a$ \\
\hline $\begin{array}{l}\text { Acidez titulável } \\
\text { (\% ácido cítrico) }\end{array}$ & $2,290,05$ & $1,040,03 a$ & $1,000,05 a b$ & $0,980,06 b$ \\
\hline Umidade (\%) & $88,670,03$ & $26,500,05 a$ & $25,670,036 a$ & $25,330,04 a$ \\
\hline Proteína (\%) & $1,820,05$ & $4,070,06 \mathrm{c}$ & $5,510,05 b$ & $6,180,08 a$ \\
\hline Cinzas (\%) & $0,660,02$ & $0,960,02 b$ & $0,940,05 b$ & $1,080,01 a$ \\
\hline Lipídeos (\%) & $0,520,09$ & $0,720,09 a$ & $0,740,08 \mathrm{a}$ & $0,760,1 \mathrm{a}$ \\
\hline Fibra $(\%)$ & $0,720,09$ & $3,430,07 a$ & $3,550,09 b$ & $3,590,05 b$ \\
\hline Vitamina C (mg.100 g) ${ }^{-1}$ & $19,232,83$ & $24,732,03 a$ & $24,092,97 a$ & $24,392,11 a$ \\
\hline Açúcares totais (\%) & $2,450,53$ & $48,020,61 \mathrm{c}$ & $45,010,55 b$ & $43,630,63 a$ \\
\hline Açúcares redutores (\%) & $6,020,06$ & $29,980,06 a$ & $27,230,05 b$ & $26,660,06 \mathrm{c}$ \\
\hline
\end{tabular}

Para as três formulações estudadas observouse boa estruturação, com percentagens de polpa em relação à massa final do produto consideradas elevadas, verificando-se teores de polpa de $81,43 \%$ para a formulação 1, 79,49\% para a formulação $2 \mathrm{e}$ $78,24 \%$ para a formulação 3. Mouquet et al. (1992) estudaram a textura de estruturados de polpa de manga desenvolvidos com alginato, nos quais foram possíveis boas estruturações empregando-se até $80 \%$ de polpa de manga.

Grizotto, Aguirre e Menezes (2005) encontraram, para estruturados de abacaxi, manga e mamão, teores de umidade de 60,80\%, 64,10\% e 82,2\% respectivamente; estes teores são considerados elevados e são resultado da não utilização da etapa de secagem durante o processamento dos estruturados elaborados por esses autores.

Os estruturados de cupuaçu apresentaram resistência ao corte, sem pegajosidade ao toque, estando inclusive abaixo da faixa de 0,65 a 0,90 de atividade de água estabelecida para alimentos de umidade intermediária (CHIRIFE; BUERA, 1994), o que garante maior segurança e estabilidade para esses produtos. Foi relatado por Grizotto et al. (2007), que a secagem favoreceu a formação de uma película externa, contribuindo para a formação de um tipo de casca que melhorou a textura final do produto, semelhante ao que foi observado para os estruturados de cupuaçu. Segundo McHugh e Huxsoll (2002), na estruturação de polpas de pêssego sem adição de gelatina o teor de água no produto final está diretamente relacionado ao teor de polpa empregado, sendo este o fator que mais interfere na estruturação e textura do produto final. Os mesmos autores ainda afirmam que produtos estruturados, uma vez submetidos à secagem, apresentam textura mais firme do que aqueles que foram apenas resfriados. A utilização de gelatina e a etapa de secagem posterior à refrigeração possibilitou a estruturação eficiente de estruturados com elevada quantidade de polpa de cupuaçu e baixos teores de umidade.

Em relação à atividade de água dos estruturados, foi possível constatar que o aumento da concentração de gelatina está diretamente relacionado à redução na atividade de água nos mesmos, provavelmente em razão das propriedades supressoras de água de tal componente (FISZMAN; DURÁN, 1992). Os valores de atividade de água observados neste trabalho variaram entre 0,55 e 0,60 e encontram-se bem próximos daqueles citados por Santos (2003), que relata valores de atividade de água de 0,58 para estruturados de manga secos em estufa, e daqueles citados por Collins e Washam-Hutsell (1987), que citam valores de 0,48 e 0,58 para estruturados de batata doce. Grizotto, Aguirre e Menezes (2005) elaboraram estruturados com pectina de baixa metoxilação e alginato de cálcio, sem a etapa de secagem, e obtiveram estruturados com elevados valores de atividade de água, tais como 0,85 a 0,93 para estruturados de abacaxi, 0,85 a 0,91 para estruturados de manga e 0,89 a 0,92 para estruturados de mamão. Esses valores são bem superiores aos observados para os estruturados de cupuaçu elaborados com gelatina, mostrados neste trabalho.

Para a acidez titulável dos estruturados foram observados valores de 1,04, 1,00 e 0,98\%, respectivamente para as formulações 1,2 e 3 . De acordo com Bellard (1995) e Santos (2003), a acidez da polpa utilizada pode interferir fortemente na geleificação dos estruturados. Já Grizotto et al. (2007) afirmam que a mistura de pectina de baixa metoxilação e alginato de cálcio permitem uma eficiente estruturação de polpas ácidas. Semelhante ao que foi observado para a polpa de cupuaçu, caracterizada como uma polpa ácida, e que demonstrou boa estruturação com a associação dos hidrocoloides utilizados para sua estruturação.

Com relação ao teor de proteína, observa-se diferença estatística entre as três formulações estudadas, devido às diferentes quantidades de gelatina (5, 8 e 10\%) empregadas nas três formulações estudadas.

Para o teor de fibras foram encontrados valores que variaram de 3,43 a 3,59\% (Tabela 1). Esse aumento se deu em razão do aumento da concentração de gelatina e maior concentração do produ- 
to final, pela associação do hidrocoloide com a água do produto. Segundo a Portaria no 27, de 13 de janeiro de 1998, que aprova o Regulamento Técnico referente à Informação Nutricional Complementar, os estruturados de cupuaçu podem ser classificados como alimentos fonte de fibras, pois apresentam valores superiores a $30 \mathrm{~g} \mathrm{~kg}^{-1}$, mínimo exigido pela legislação para alimentos incluídos nessa categoria.

Quanto à análise de vitamina $\mathrm{C}$, observou-se que o valor médio encontrado para os estruturados de cupuaçu, $240 \mathrm{mg} \mathrm{kg}^{-1}$, é bastante significativo quando comparado aos teores observados para outras frutas in natura, como carambola (239,6 mg $\mathrm{kg}^{-1}$ ), manga Haden (174 $\mathrm{mg} \mathrm{kg}^{-1}$ ), cajá-manga (267 mg kg-1) e caqui (296 mg kg-1) (TEIXEIRA, 2001; TACO, 2006).

As três formulações de estruturados de cupuaçu mostraram-se bem aceitas em relação a todos os atributos avaliados na análise sensorial, com notas variando de 6,64 a 8,37, que corresponde a valores de gostei ligeiramente e gostei muitíssimo (Tabela 2). Observou-se que a Formulação 3, com 10\% de gelatina, apresentou médias superiores para todos os atributos avaliados, indicando a preferência dos provadores por estruturados mais firmes e consistentes.

Observa-se que as Formulações 2 e 3 destacaram-se no nível de certeza dos provadores com relação à intenção de compra, com mais de $90 \%$ de seus provadores confirmando que "possivelmente" ou "certamente" comprariam o produto se o encontrassem à venda (Figura 2).

Tabela 2 - Médias das notas atribuídas pelos provadores para a aceitação sensorial das formulações de estruturado de cupuaçu.

\begin{tabular}{lccccc}
\hline \multirow{2}{*}{ Estruturados } & \multicolumn{5}{c}{ Notas atribuídas } \\
\cline { 2 - 6 } & Aparência & Aroma & Textura & Sabor & Impressão global \\
\hline Formulação 1 & $8,10 \mathrm{a}$ & $7,80 \mathrm{a}$ & $7,32 \mathrm{~b}$ & $7,70 \mathrm{~b}$ & $7,42 \mathrm{a}$ \\
Formulação 2 & $7,29 \mathrm{~b}$ & $6,64 \mathrm{~b}$ & $7,50 \mathrm{~b}$ & $7,69 \mathrm{~b}$ & $7,29 \mathrm{a}$ \\
Formulação 3 & $8,37 \mathrm{a}$ & $7,95 \mathrm{a}$ & $8,00 \mathrm{a}$ & $8,10 \mathrm{a}$ & $7,59 \mathrm{a}$ \\
\hline
\end{tabular}

Médias com letras iguais, na mesma coluna, não diferem entre si pelo Teste de Tukey, a 5\% de probabilidade.

Quanto à impressão global, verificou-se 82,4\% de aceitação para a formulação $1,81 \%$ para a formulação 2 e 84,3\% para a formulação 3 , confirmando a boa aceitação dos produtos pelos potenciais consumidores.

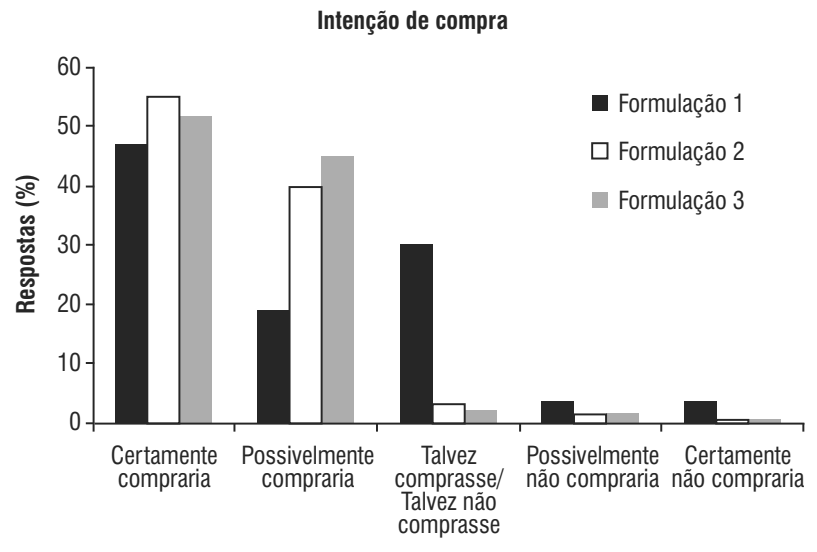

Figura 2. Frequência dos valores hedônicos atribuídos à intenção de compra dos estruturados de cupuaçu.

\section{Conclusões}

Os resultados mostram que é possível estabelecer um procedimento tecnológico para a produção de fruta estruturada com polpa de cupuaçu. A associação de gelatina e pectina proporcionou a obtenção de géis de polpa estáveis, firmes e sem pegajosidade durante o toque.

A secagem mostrou-se eficiente na produção de frutas estruturadas de reduzida atividade de água, permitindo a obtenção de produtos estáveis sob o ponto de vista microbiológico, haja vista que os valores de atividade de água encontrados qualificam todas as formulações de estruturados de cupuaçu como alimentos de baixa atividade de água.

Todas as formulações apresentaram médias de aceitação elevadas, com destaque para a formulação 3 , que, além de bem aceita, demonstrou maiores teores de proteína, fibra e vitamina $\mathrm{C}$, o que indica o potencial de consumo do produto na forma em que se apresenta, além de ser considerado um produto conveniente e saudável.

\section{Referências}

ALZAMORA, S.M.; ARGAIZ, A.; WELTI, J. Fruit preservation by combined factors. Food Research International, Oxford, v.25, n.2, p.159-165, 1992.

AOAC. (Associaton of Official Analytical Chemists). Official methods of analysis. 14. ed. Arlington: AOAC, 1984. 1141p.

Official methods of analysis of the Association of Official Analytical Chemists. 16 ed. Washington: Horwitz, W, 1997. 
BELLARD, F.B. Utilização de alginato de sódio na produção de umgel estruturado de suco de maracujá (Passiflora edulis S), 1995, 60f. Dissertação (Mestrado em Tecnologia de Alimentos), Faculdade de Engenharia de Alimentos, Universidade Estadual de Campinas, 1995.

BENASSI, M. T.Análise dos efeitos de diferentes parâmetros de vitamina Cem vegetais processados, 1990. 159f. Dissertação (Mestrado em Tecnologia de Alimentos). Faculdade de Engenharia de Alimentos, Universidade Estadual de Campinas. Campinas, 1990.

BLIGH, E.G.; DYER, W.J. A rapid method of total lipid and purification. Canadian Journal Biochemistry Physical, v.37, p. 911-917, 1959.

BRASIL. MINISTÉRIO DA SAÚDE. Portaria no 27, de 13 de janeiro de 1998. Aprova o Regulamento Técnico referente à Informação Nutricional Complementar (declarações relacionadas ao conteúdo de nutrientes), constantes do anexo desta Portaria. Diário Oficial da União. Brasília, Secretaria de Vigilância Sanitária do Ministério da Saúde, 16 de janeiro de 1998.

BUENO, S. M.; GRACIANO, R. A. S.; FERNANDES, E. C. B.; GARCIA-CRUZ, C. H. Avaliação da qualidade de polpas de frutas congeladas. Revista do Instituto Adolfo Lutz, São Paulo, v.62, n.2, p.121-126, 2002.

CALZAVARA, B.B.G.; MIILLER, C. H.; KAHNAGE, O.N.C. Fruticultura tropical: o cupuaçuzeiro. Belém: Embrapa/Cpatu, 1987.99p.

CHIRIFE, J.; BUERA, M.D.P. Water activity, glass transition and microbial stability in concentraded/semimois food systems. Journal of Food Science, v.59, n.5, p.925-927, 1994.

COLLINS, J.L.; WASHAM-HUTSELL, L. Physical, chemical, sensory and microbiology attributes of sweet potato leather. Journal of Food Science, v.52, n.3, p.646648, 1987.

COSTA, M.C.; MAIA, G.A.; FILHO, M.S.M.S.; FIGUEIREDO, R.W.; NASSU, R.T.; MONTEIRO, J.C.S. Conservação de polpa de cupuaçu [Theobroma grandiflorum (Willd. Ex Spreng.) Schum] por métodos combinados. Revista Brasileira de Fruticultura, v.25, n.2, p.213-215, 2003.

FIZMAN, S.M. Características de los alimentos estructurado y reestructurado, uso de los hydrocolloides in las formulaciones para calentamiento con microondas. In: FERREIRA, V.L.P.; SOLER, M.P. (Coords.). Curso sobre as propriedades de hidrocoloides e aplicações. Campinas: Ital, 1992. p.1-3.

FIZMAN, S.M.; DURAN, L. Effect of fruit pulps and sucrose on the compression response of different polysaccharides gel systems. Carbohydrate Polymers, Valencia, v.17, n.1, p.11-17, 1992.

FREITAS, S.M.L. Utilização de alginato de sódio em texturizados de suco misto de laranja e cenoura de valor energético reduzido. 1999, 110f. Dissertação (Mestrado em Tecnologia de Alimentos). Faculdade de Engenharia de Alimentos, Universidade Estadual de Campinas, Campinas, 1999.

FREIRE, M.T.A.; PETRUS, R.R.; FREIRE, C.M.A.; OLIVEIRA, C.A.F.; FELIPE, A.M.P.F.; GATTI, J.B. Caracterização físico-química, microbiológica e sensorial de polpa de cupuaçu congelada (Theobroma grandiflorum Schum). Brazilian Journal of Food Technology, São Paulo, v.12, n.1, p.9-16, 2009.

GOERING, H.K.; VAN SOEST, P.J. Forage fiber analysis (Apparatus, reagents, procedures and some applications). Agricultural Handbook, Washington: Agricultural Research Service, 1970. p.19

GRIZOTTO, R.K.; AGUIRRE, J.M.; MENEZES, H.C. Frutas estruturadas de umidade intermediária obtidas de polpas concentradas de abacaxi, manga e mamão. Ciência e Tecnologia de Alimentos, Campinas, v.25, n.1, p.158-164, 2005.

GRIZOTTO, R.K.; BERBARI, S.A.G.; MOURA, S.C.S.R.; CLAUS, M.L. Estudo da vida-de-prateleira de fruta estruturada e desidratada obtida de polpa concentrada de mamão, Ciência e Tecnologia de Alimentos, Campinas, v.26, n.3, p.709-714, 2006.

GRIZOTTO, R.K.; BRUNS, R.E.; AGUIRRE, J.M.; MENEZES, H.C. Optimizing restructuring process of concentrated pineapple pulp using Response Surface Methodology. In: INTERNATIONAL CONGRESS ON ENGINEERINGAND FOOD, 2004, Montpellier. Anais... Montpellier, 2004, p.1-5.

GRIZOTTO, R.K.; BRUNS, R.E.; AGUIRRE, J.M.; MENEZES, H. C. Technolgical aspects of the restructuring of concentrated pineapple pulp. Food Science and Technology, London, v.40, n.1, p.759-765, 2007.

LANE, J.H.; EYNON, L. Determination of reducing sugars by Fehling's solution with methylene blue indicator. Normam Rodge, London, 8 p., 1934.

LOPES, A.S. Estudo químico e nutricional de amêndoas de cacau (Theobroma cacao L.) e cupuaçu (Theobroma grandiflorum Schum) em função do processamento. 2000, 112f. Dissertação (Mestrado em Tecnologia de Alimentos), Faculdade de Engenharia de Alimentos, Universidade Estadual de Campinas, Campinas, 2000.

MCHUGH,T. H.; HUXSOLL, C.C. Extrusion processing of restructured peach and peach/starch gels. LebensmittelWissenschaft und-Technologie, v.32, n. 8, p. 513-520. 2002.

MINISTÉRIO DA AGRICULTURA E DO ABASTECIMENTO. Instrução Normativa ${ }^{\circ} 1$, de 07 de janeiro de 2000. Aprova o Regulamento Técnico para fixação dos Padróes de Identidade e Qualidade para polpa de fruta. Diário Oficial da União, Brasília-DF, Seção I, p.54.

MOUQUET, C.; DUMA, J.C.; GUILBERT, S. Texturization of sweetened mango pulp: optimization using response surface methodology. Journal Food Science, v.57, n.6, p.1395-1400, 1992.

SANTOS, C.N.P. Elaboração de um estruturado de polpa de manga (Mangifera indica. Cv Tommy Atkins) parcial- 
mente desidratada por osmose. Campinas, 2003. Dissertação (Mestrado em Tecnologia de Alimentos) - Faculdade de Engenharia de Alimentos, Universidade Estadual de Campinas, Campinas, 2003.

STONE, H.; SIDEL, J.L. Affective testing. In: Sensory evaluations practices. New York: Academic Press, 1985. p.227-252.

TACO. Tabela brasileira de composição de alimentos. Versão II. 2ed. Campinas, SP, Nepa - Unicamp, 2006. 113p. Disponível em: <www.unicamp.br/nepa/taco/>. Acesso em: 21 agosto 2009.
TEIXEIRA, G.H.A.; DURIGAN, J.F.; DONADIO, L.C.; DA SIIVAJ.A.A. da. Caracterização pós-colheita de seis cultivares de carambola (averrhoa carambola 1.). Revista Brasileira de Fruticultura, Jaboticabal, v.23, n. 3, p.1-7, 2001.

VIJAYANAND, P.; YADAV,A. R; BALASUBRAMANYAM, N.; NARASIMHAM, P. Storage stability of guava fruit bar prepared using a new process. Lebensmittel-Wissenschaft und-Technologie, v.33, n.1, p.132-137, 2000.

VILLACHICA, H. Frutales y hortalizas promisorios de la Amazônia. Lima: Tratado de Cooperacion Amazônica, 1996. 385p. 